\title{
$1 \quad$ Intelligible speech synthesis from neural decoding of spoken sentences
}

2 Authors Gopala K. Anumanchipalli ${ }^{1,2} *$, Josh Chartier ${ }^{1,2,3 *}$, Edward F. Chang ${ }^{1,2,3}$

$3 *$ Authors contributed equally

4

13 Correspondence and requests for materials should be addressed to

\section{Edward.Chang@ucsf.edu}

15 The authors declare no competing interests.

\section{Abstract}

18 The ability to read out, or decode, mental content from brain activity has significant

19 practical and scientific implications ${ }^{1}$. For example, technology that translates cortical

20 activity into speech would be transformative for people unable to communicate as a result

21 of neurological impairment ${ }^{2,3,4}$. Decoding speech from neural activity is challenging

22 because speaking requires extremely precise and dynamic control of multiple vocal tract

23 articulators on the order of milliseconds. Here, we designed a neural decoder that

24 explicitly leverages the continuous kinematic and sound representations encoded in

25 cortical activity ${ }^{5,6}$ to generate fluent and intelligible speech. A recurrent neural network

26 first decoded vocal tract physiological signals from direct cortical recordings, and then

27 transformed them to acoustic speech output. Robust decoding performance was achieved

28 with as little as 25 minutes of training data. Naïve listeners were able to accurately 
29 identify these decoded sentences. Additionally, speech decoding was not only effective

30 for audibly produced speech, but also when participants silently mimed speech. These

31 results advance the development of speech neuroprosthetic technology to restore spoken

32 communication in patients with disabling neurological disorders.

\section{Text}

35 Neurological conditions that result in the loss of communication are devastating.

36 Many patients rely on alternative communication devices that measure residual nonverbal

37 movements of the head or eyes ${ }^{7}$, or even direct brain activity ${ }^{8,9}$, to control a cursor to

38 select letters one-by-one to spell out words. While these systems dramatically enhance a

39 patient's quality of life, most users struggle to transmit more than 10 words $/$ minute $^{10}$, a

40 rate far slower than the average of 150 words/min in natural speech. A major hurdle is

41 how to overcome the constraints of current spelling-based approaches to enable far higher

42 communication rates.

43 A promising alternative to spelling-based approaches is to directly synthesize

$44 \operatorname{speech}^{11,12}$. Spelling is a sequential concatenation of discrete letters, whereas speech is

45 produced from a fluid stream of overlapping, multi-articulator vocal tract movements ${ }^{13}$.

46 For this reason, a biomimetic approach that focuses on vocal tract movements and the

47 sounds they produce may be the only means to achieve the high communication rates of

48 natural speech, and likely the most intuitive for users to learn ${ }^{14,15}$. In patients with

49 paralysis, for example from ALS or brainstem stroke, high fidelity speech control signals

50 may only be accessed by directly recording from intact cortical networks using a brain-

51 computer interface. 
52 Our goal was to demonstrate the feasibility of a neural speech prosthetic by

53 translating brain signals into intelligible synthesized speech at the rate of a fluent speaker.

54 To accomplish this, we recorded high-density electrocorticography (ECoG) signals from

55 three participants undergoing intracranial monitoring for epilepsy treatment as they spoke

56 several hundred sentences aloud. We designed a recurrent neural network that decoded

57 cortical signals with an explicit intermediate representation of the articulatory dynamics

58 to generate audible speech.

59 An overview of our two-stage decoder approach is shown in Figure 1a-d. In the

60 first stage, a bidirectional long short term memory (bLSTM) recurrent neural network ${ }^{16}$

61 decodes articulatory kinematic features from continuous neural activity (Figure 1a, b). In

62 the second stage, a separate bLSTM decodes acoustic features from the decoded

63 articulatory features from stage 1 (Figure 1c). The audio signal is then synthesized from

64 the decoded acoustic features (Figure 1d).

65 There are three sources of data for training the decoder: high density ECoG

66 recordings, acoustics, and articulatory kinematics. For ECoG, high-gamma amplitude

67 envelope $(70-200 \mathrm{~Hz})^{17}$, and low frequency component $(1-30 \mathrm{~Hz})^{18}$ were extracted from

68 the raw signal of each electrode. Electrodes were selected if they were located on key

69 cortical areas for speech: ventral sensorimotor cortex (vSMC) ${ }^{19}$, superior temporal gyrus

$70(\mathrm{STG})^{20}$, or inferior frontal gyrus (IFG) ${ }^{21}$ (Figure 1a). For acoustics, instead of a typical

71 spectrogram, we used 25 mel-frequency cepstral coefficients (MFCCs), 5 sub-band

72 voicing strengths for glottal excitation modelling, pitch, and voicing (32 features in all).

73 These acoustic parameters are specifically designed to emphasize perceptually relevant

74 acoustic features while maximizing audio reconstruction quality ${ }^{22}$. 
75 Lastly, a key component of our decoder is an intermediate articulatory kinematic

76 representation between neural activity and acoustics (Figure 1b). Our previous work

77 demonstrated that articulatory kinematics is the predominant representation in the

$78 \mathrm{vSMC}^{6}$. Since it was not possible to record articulatory movements synchronously with

79 neural recordings, we used a statistical speaker-independent Acoustic-to-Articulatory

80 inversion method to estimate vocal tract kinematic trajectories corresponding to the

81 participant's produced speech acoustics. We added additional physiological features (e.g.

82 manner of articulation) to complement the kinematics and optimized these values within

83 a speech autoencoder to infer the full intermediate articulatory kinematic representation

84 that captures vocal tract physiology during speech production (see methods). From these

85 features, it was possible to accurately reconstruct the speech spectrogram (Figure 1e,f). 

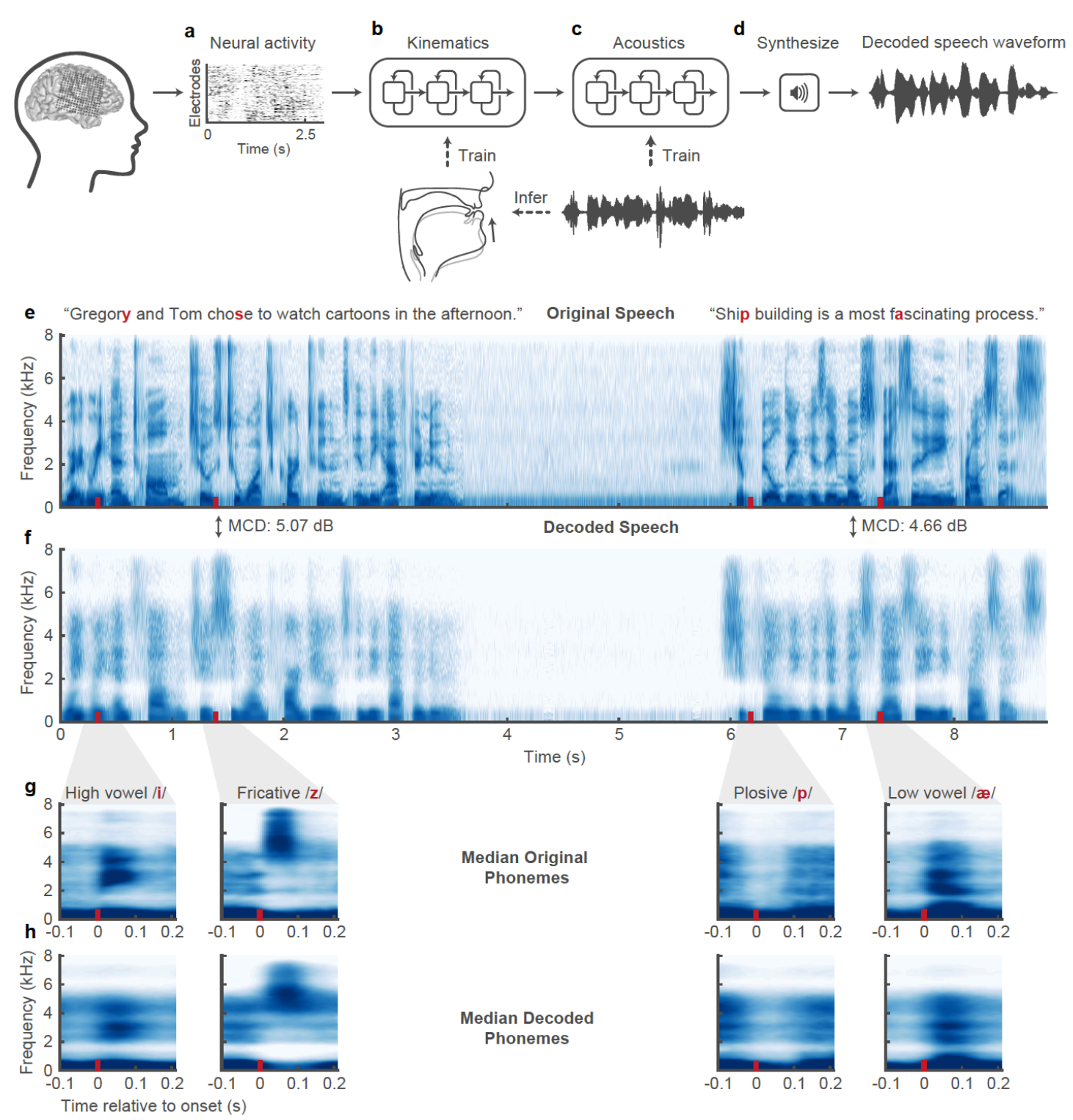

Figure 1: Speech synthesis from neurally decoded spoken sentences. a, The neural

88 decoding process begins by extracting high-gamma amplitude $(70-200 \mathrm{~Hz})$ and low-

89 frequency $(1-30 \mathrm{~Hz}) \mathrm{ECoG}$ activity. b, A 3-layer bi-directional long short term memory

90 (bLSTM) neural network learns to decode kinematic representations of articulation from

91 filtered ECoG signals. c, An additional 3-layer bLSTM learns to decode acoustics from

92 the previously decoded kinematics. Acoustics are represented as spectral features (e.g.

93 Mel-frequency cepstral coefficients (MFCCs)) extracted from the speech waveform. d,

94 Decoded signals are synthesized into an acoustic waveform. e, Spectrogram shows the

95 frequency content of two sentences spoken by a participant. f, Spectrogram of

96 synthesized speech from brain signals recorded simultaneously with the speech in e. Mel-

97 cepstral distortion (MCD), a metric for assessing the spectral distortion between two

98 audio signals, was computed for each sentence between the original and decoded audio.

99 g,h $300 \mathrm{~ms}$ long, median spectrograms that were time-locked to the acoustic onset of

100 phonemes from original (g) and decoded (h) audio. Medians were computed from 
101 phonemes in 100 sentences that were withheld during decoder training $(\mathrm{n}: / \mathrm{i} /=112, / \mathrm{z} /=$ $102115, / \mathrm{p} / 69, / \mathrm{ae} /=86)$. These phonemes represent the diversity of spectral features.

103 Original and decoded median phoneme spectrograms were well correlated $(\mathrm{r}>0.9$ for all 104 phonemes, $\mathrm{p}=1 \mathrm{e}-18$ )

105

106 Synthesis performance

107 Overall, we observed highly detailed reconstructions of speech decoded from

108 neural activity alone (See supplemental video). Examples of decoding performance are

109 shown in Figure $1(\mathrm{e}, \mathrm{f})$, where the audio spectrograms from two original spoken

110 sentences are plotted above those decoded from brain activity. The first sentence is

111 representative of the median performance and the second shows one of the best decoded

112 sentences. The decoded spectrogram contained salient energy patterns present in the

113 original spectrogram.

114 To illustrate the quality of reconstruction at the phonetic level, we compared

115 median spectrograms of phonemes from original and decoded audio. As shown in Figure

$1161 \mathrm{~g}, \mathrm{~h}$, the formant frequencies (F1-F3, seen as high energy resonant bands in the

117 spectrograms) and distribution of spectral energy for high and low vowels (/i/ and /ae/,

118 respectively) of the decoded examples closely resembled the original speech. For alveolar

119 fricatives $(/ \mathrm{z} /)$ the high frequency $(>4 \mathrm{kHz})$ acoustic energy was well represented in both

120 spectrograms. For plosives (/p/), the short pause (relative silence during the closure)

121 followed by a broadband burst of energy (after the release) was also well decoded. The

122 decoder also correctly reconstructed the silence in between the sentences when the

123 participant was not speaking.

124 To quantify performance, we tested the neural decoder for each participant on 100

125 sentences that were withheld during the training and optimization of the full model. In 
126 traditional speech synthesis, the spectral distortion of synthesized speech from ground-

127 truth is commonly reported using the mean Mel-Cepstral Distortion (MCD) ${ }^{23}$. The use of

128 Mel-Frequency bands emphasizes the distortion of perceptually relevant frequency bands

129 of the audio spectrogram ${ }^{24}$. In Figure $2 \mathrm{a}$, the MCD of neurally decoded speech was

130 compared with reference synthesis from articulatory kinematics and chance-level

131 decoding (lower MCD is better). The reference synthesis acts as a bound for performance

132 as it simulated what perfect neural decoding of the kinematics would achieve. For our

133 participants (P1, P2, P3), the median MCD scores of decoding speech were $5.14 \mathrm{~dB}, 5.55$

$134 \mathrm{~dB}$, and $5.49 \mathrm{~dB}$, all better than chance-level decoding $(\mathrm{p}<1 \mathrm{e}-18, \mathrm{n}=100$ sentences,

135 Wilcoxon signed-rank test (WSRT), for each participant). These scores were on par with

136 state-of-the-art approaches to decode speech from facial surface electromyography

137 (EMG) with similarly sized datasets (average MCD of $5.21 \mathrm{~dB})^{25}$.

138 To assess the perceptual intelligibility of the decoded speech, we used Amazon

139 Mechanical Turk to evaluate naïve listeners' ability to understand the neurally decoded

140 trials. We asked 166 people to identify which of 10 sentences (written on screen)

141 corresponded to the decoded audio they heard. The median percentage of participants

142 who correctly identified each sentence was $83 \%$, significantly above chance $(10 \%)$

143 (Figure 2b).

144 In addition to spectral distortion and intelligibility, we also examined the

145 correlations between original and decoded spectral features. The median correlations (of

146 sentences, Pearson's r) of the mean decoded spectral feature (pitch +25 MFCCs +

147 excitation strengths + voicing) for each participant were $0.55,0.49$, and 0.42 (Figure 2c).

148 Similarly, for decoded kinematics (the intermediate representation), the median 
149 correlations were $0.66,0.54$, and 0.50 (Figure 2d). Finally, we examined three key

150 aspects of prosody for intelligible speech: pitch (f0), speech envelope, and voicing ${ }^{26}$

151 (Figure 2d). For all participants, these features were decoded well above chance-level

152 correlations ( $r>0.6$, except f0 for P2: $r=0.49, p<1 e-10, n=100$, WSRT, for all

153 participants and features in Figure 2c-d). Correlation decoding performance for all other

154 features is shown in Extended Data Figure 1a,b.
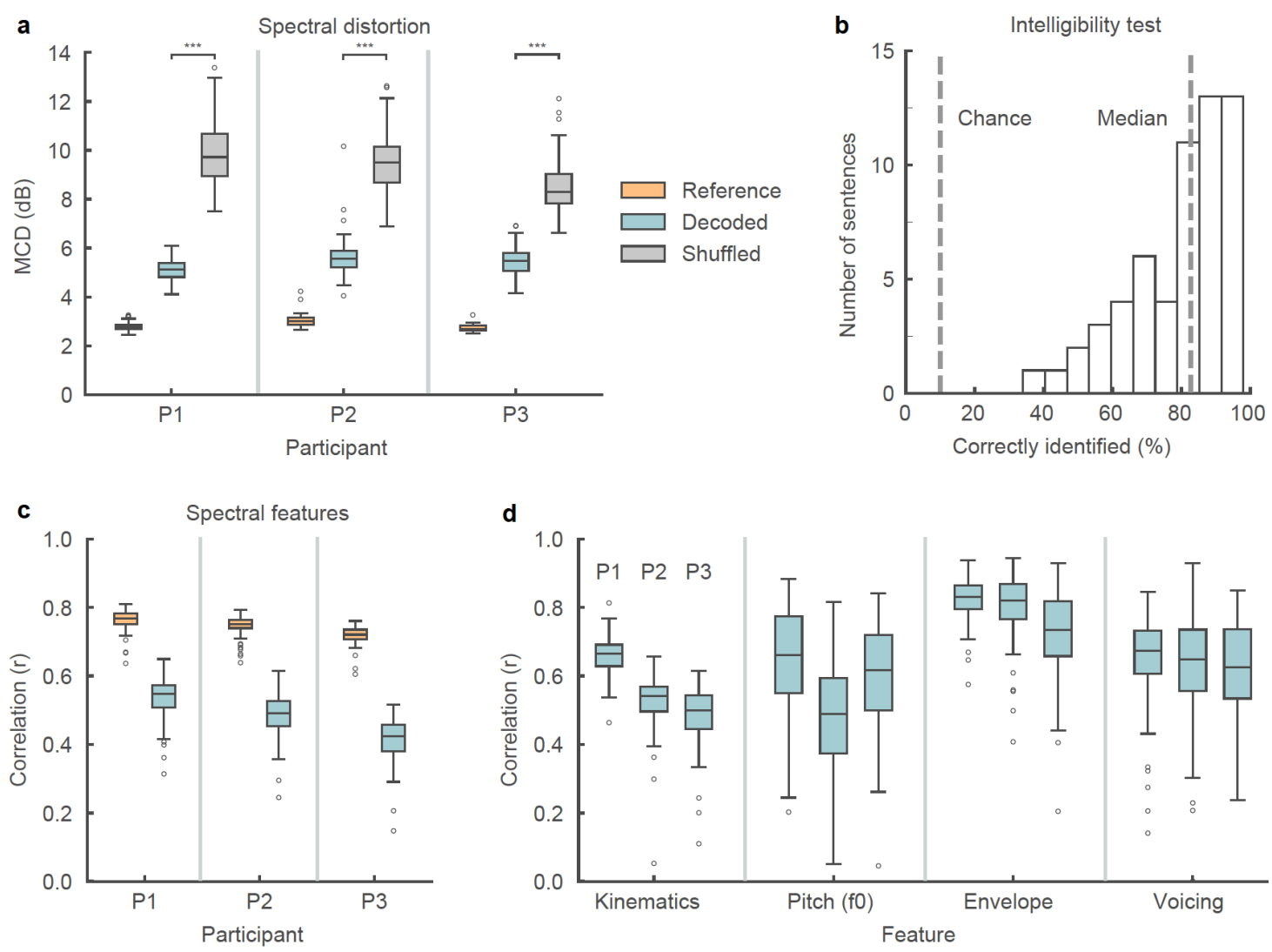

156 Figure 2: Decoded speech intelligibility and feature-specific performance.. a,

157 Spectral distortion, measured by Mel-Cepstral Distortion (MCD) (lower values are

158 better), between original spoken sentences and neurally decoded sentences that were held

159 out from model training $(\mathrm{n}=100)$. Reference MCD refers to the MCD resulting from the

160 synthesis of original kinematics without neural decoding and provides an upper bound for

161 performance. MCD scores were compared to chance-level MCD scores obtained by

162 shuffling data before decoding. b, Decoded sentence intelligibility was assessed by

163 asking naïve participants to identify the sentence they heard from 10 choices. Each

164 sample $(n=60)$ represents the percentage of correctly identified trials for one sentence.

165 The median sentence was correctly identified $83 \%$ of the time. c, Correlation of original 
166 and decoded spectral features. Values represent the mean correlation of the 32 spectral

167 features for each sentence $(n=100)$. Correlation performance for individual spectral

168 features is reported in extended data figure $1 \mathrm{~b}$. d, Correlations between original and

169 decoded intelligibility-relevant features. Kinematic values represent the mean correlation

170 of the 33 kinematic features (the intermediate representation) for each sentence $(n=100)$.

171 Correlation performance for individual kinematic features is reported in extended data

172 figure 1a. Box plots depict median (horizontal line inside box), 25th and 75th percentiles

173 (box), 25/75th percentiles $\pm 1.5 \times$ interquartile range (whiskers), and outliers (circles).

174 Distributions were compared with each as other as indicated or with chance-level

175 distributions using two-tailed Wilcoxon signed-rank tests ( $\mathrm{p}<1 \mathrm{e}-10, \mathrm{n}=100$, for all

176 tests).

177

179 Effects of model design decisions

180 The following analyses were performed on data from P1. In designing a neural

181 decoder for clinical applications, there are several key considerations regarding the input

182 to the model. First, in patients with severe paralysis or limited speech ability, training

183 data may be very difficult to obtain. In audio-based commercial applications like digital

184 assistants, successful speech synthesis from text relies on tens of hours of speech $^{27}$.

185 Despite having limited neural data, we observed high decoding performance, and

186 therefore we wanted to assess how much data was necessary to achieve this level of

187 performance. Furthermore, we wanted to see if there was a clear advantage in explicitly

188 modeling articulatory kinematics as an intermediate step over decoding acoustics directly

189 from the ECoG signals. The motivation for including articulatory kinematics was to

190 reduce the complexity of the ECoG-to-acoustic mapping because it captures the

191 physiological process by which speech is generated and is encoded in the $\mathrm{vSMC}^{6}$.

192 We found robust performance could be achieved with as little as 25 minutes of

193 speech, but performance continued to improve with the addition of more data (Figure

194 3a,b). A crucial factor in performance was the articulatory intermediate training step. 
195 Without this step, direct ECoG to acoustic decoding MCD was offset by $0.54 \mathrm{~dB}$ using

196 the full data set (Figure 3a) $(\mathrm{p}=1 \mathrm{e}-17, \mathrm{n}=100$, WSRT), a substantial difference given that

197 a change in MCD as small as $0.2 \mathrm{~dB}$ is perceptually noticeable ${ }^{28}$. While the two

198 approaches might perform comparably with enough data, the biomimetic approach using

199 an intermediate articulatory representation is superior because it requires less training

200 data.

201 Second, we wanted to understand the acoustic-phonetic properties that were

202 preserved in decoded speech because they are important for relative phonetic

203 discrimination. To do this, we compared the acoustic properties of decoded phonemes to

204 ground truth by constructing a statistical distribution of the spectral feature vectors for

205 each phoneme. Using Kullback-Leibler (KL) divergence, we compared the distribution of

206 each decoded phoneme to the distribution of each ground-truth phoneme to determine

207 how similar they were (Figure 3c). From the acoustic similarity matrix of only ground-

208 truth phoneme-pairs (Extended Data Figure 2), we expected that, in addition to the same

209 decoded and ground-truth phoneme being similar to one another, phonemes with shared

210 acoustic properties would also be characterized as similar to one another. For example,

211 two fricatives will be more acoustically similar to one another than to a vowel.

212 Hierarchical clustering on the KL-divergence of each phoneme pair demonstrated

213 that phonemes were clustered into four main groups. These groups represent the primary

214 decoded acoustic differences between phonemes. Within each group, phonemes were

215 more likely to be confused with one another due to their shared acoustic properties. For

216 instance, a decoded /s/ may easily be confused with /z/ or other phonemes in Group 1.

217 Group 1 contained consonants with an alveolar place of constriction. Group 2 contained 
218 almost all other consonants. Group 3 contained mostly high vowels. Group 4 contained

219 mostly mid and low vowels. The difference between groups tended to correspond to

220 variations along acoustically significant dimensions (frequency range of spectral energy

221 for consonants, and formants for vowels). These groupings were similar to those obtained

222 by clustering KL-divergence of ground-truth phoneme pairs (Extended Data Figure 2).

223 Third, since the success of the decoder depends on the initial electrode placement,

224 we wanted to assess how much the cortical activity of each brain region contributed to

225 decoder performance. We quantified the contributions of the vSMC, STG, and IFG by

226 training decoders in a leave-one-region-out fashion and comparing performance (Figure

227 3d). Removing any region led to decreased decoder performance (Figure 3e-f) ( $<<3 e-4$,

$228 \mathrm{n}=100$, WSRT). However, excluding vSMC resulted in the largest decrease in

229 performance (1.13 dB MCD increase). 

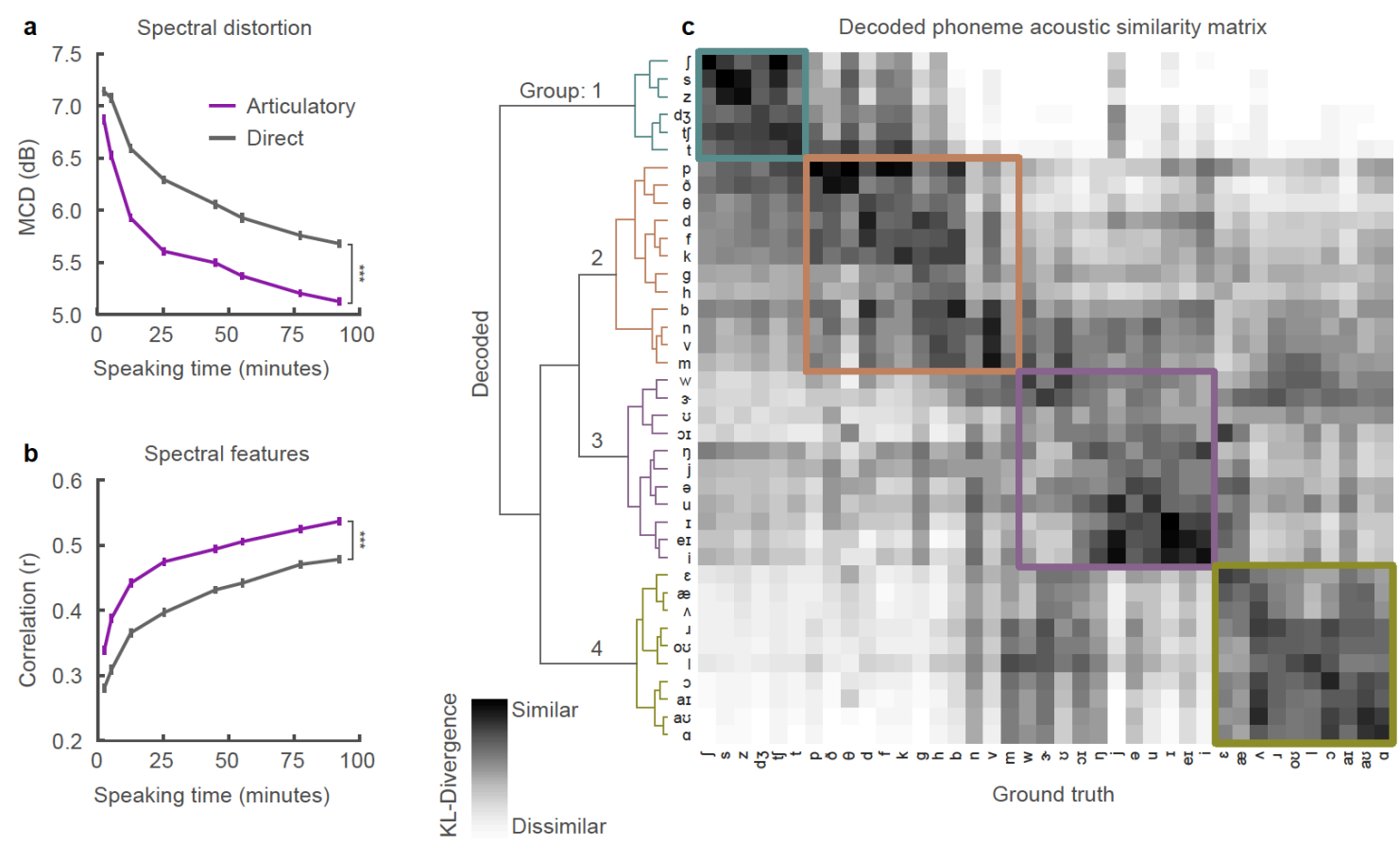

d

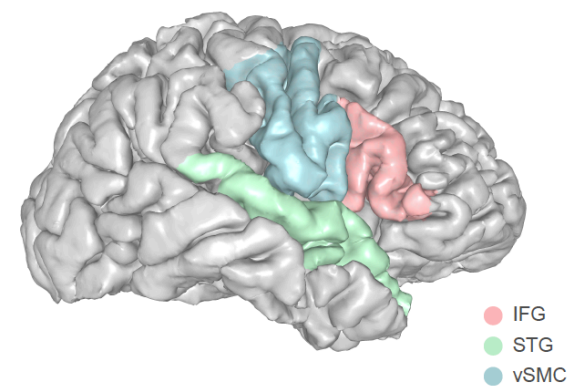

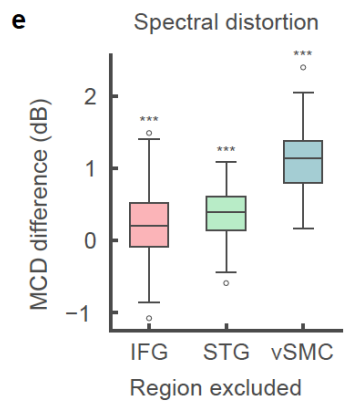

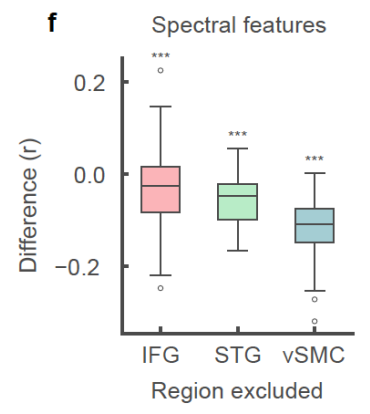

231 Figure 3: Effects of model design decisions. a, b, Mean correlation of original and 232 decoded spectral features (a) and mean spectral distortion (MCD) (b) for model trained on varying amounts of training data. Training data was split according to recording session boundaries resulting the following sizes: $2.4,5.2,12.6,25.3,44.9,55.2,77.4$, and 92.3 minutes of speaking data. The neural decoding approach that included an articulatory intermediate stage (purple) performed significantly better with every size of training data than direct ECoG to acoustics decoder (grey) (all: $\mathrm{p}<1 \mathrm{e}-5, \mathrm{n}=100$; Wilcoxon signed-rank test, error bars $=\mathrm{SE}$ ). c, Acoustic similarity matrix compares acoustic properties of decoded phonemes and originally spoken phonemes. Similarity is computed by first estimating a gaussian kernel density for each phoneme (both decoded and original) and then computing the Kullback-Leibler (KL) divergence between a pair of decoded and original phoneme distributions. Each row compares the acoustic properties of a decoded phoneme with originally spoken phonemes (columns). Hierarchical clustering was performed on the resulting similarity matrix. d, Anatomical reconstruction of a single participant's brain with the following regions used for neural decoding: ventral sensorimotor cortex (vSMC), superior temporal gyrus (STG), and inferior frontal gyrus (IFG). e, f, Difference in spectral distortion (MCD) (e), and difference in 
248 correlation (Pearson's r) performance (f) between decoder trained on all regions and

249 decoders trained on all-but-one region. Exclusion of any region resulted in decreased 250 performance $(\mathrm{p}<3 \mathrm{e}-4, \mathrm{n}=100$; Wilcoxon signed-rank test). Box plots as described in 251 Figure 2.

253 Silently mimed speech decoding

254 Finally, since future speech decoding applications must work even when speakers

255 do not produce audible sounds, we tested our decoder with a held-out set of 58 sentences

256 in which the participant (P1) audibly produced each sentence and then mimed the same

257 sentence, making the same kinematic movements but without making sound. Even

258 though the decoder was not trained on any mimed speech, the spectrograms of

259 synthesized silent speech demonstrated similar spectral features when compared to

260 synthesized audible speech of the same sentence (Figure 4a-c). After dynamic time

261 warping the acoustics of the decoded silent speech with the original audio of the

262 preceding audibly produced sentence, we calculated the spectral distortion and

263 correlation of the spectral features (Figure 4d,e). As expected, performance on mimed

264 speech was inferior to spoken speech (30\% MCD difference) although this is consistent

265 with earlier work on silent facial EMG-to-speech synthesis where decoding performance

266 from EMG signals was significantly worse when participants silently articulated without

267 audible speech output ${ }^{29}$. The performance gap may also be due to the absence of voicing

268 and laryngeal activation. This demonstrates that it is possible to decode important

269 spectral features of speech that were never audibly uttered $(\mathrm{p}<1 \mathrm{e}-11$, compared to

270 chance, $\mathrm{n}=58$; Wilcoxon signed-rank test). 
bioRxiv preprint doi: https://doi.org/10.1101/481267; this version posted November 29, 2018. The copyright holder for this preprint (which was not certified by peer review) is the author/funder, who has granted bioRxiv a license to display the preprint in perpetuity. It is made available under aCC-BY-NC-ND 4.0 International license.

a "Is this seesaw safe?"

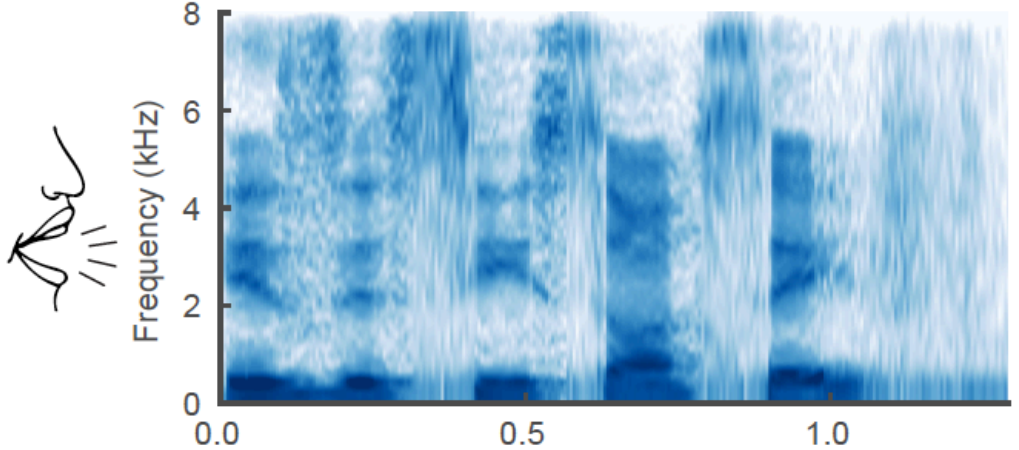

b

Decoded (Audible)

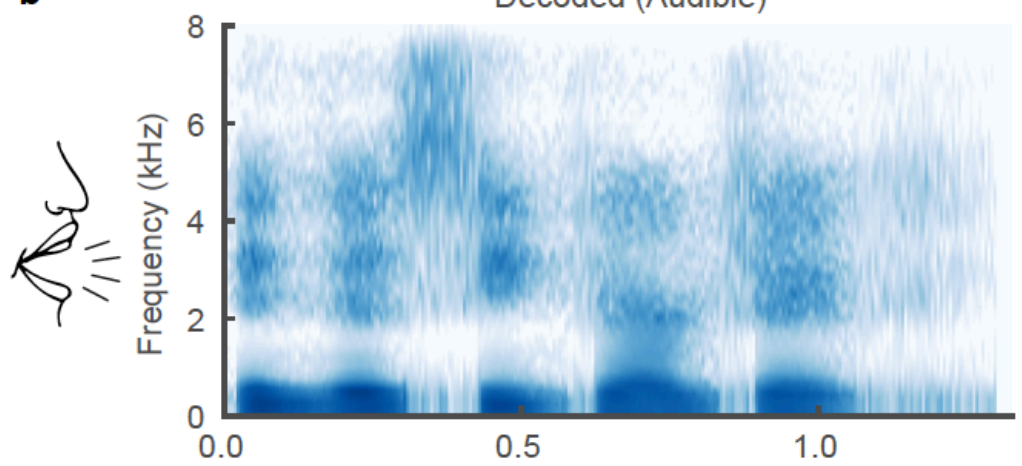

C Decoded (Mimed)
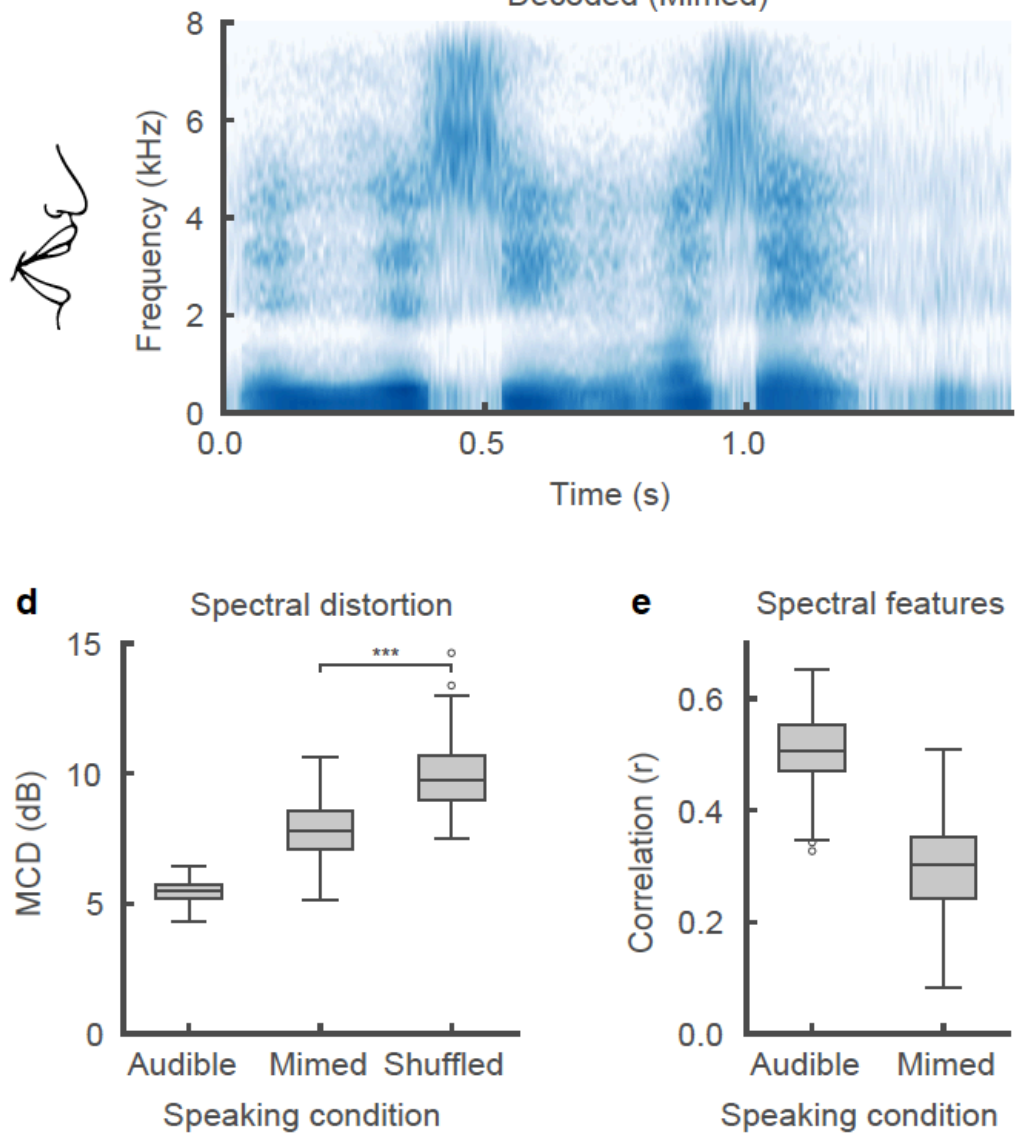

e Spectral features

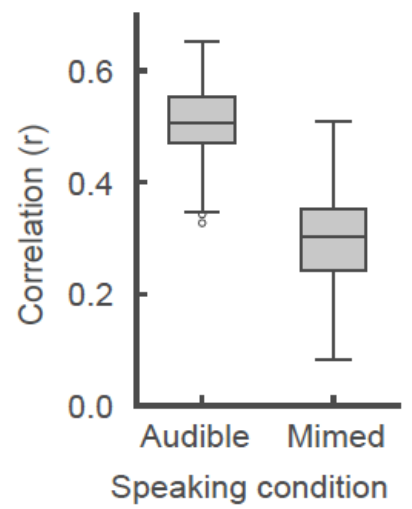


272 Figure 4: Speech synthesis from neural decoding of silently mimed speech. a-c,

273 Spectrograms of original spoken sentence (a), neural decoding from audible production

274 (b), and neural decoding from silently mimed production (c). d, e, Spectral distortion

275 (MCD) (d) and correlation of original and decoded spectral features (e) for audibly and

276 silently produced speech. Since correlations are with respect to original audibly produced

277 sentences, decoded sentences that were silently mimed were dynamically time-warped

278 according to their spectral features. Decoded sentences were significantly better than

279 chance-level decoding for both speaking conditions $(\mathrm{p}<1 \mathrm{e}-11$, for all comparisons, $\mathrm{n}=$

280 58; Wilcoxon signed-rank test). Box plots as described in Figure 2.

\section{Discussion}

Our results demonstrate intelligible speech synthesis from ECoG during both

284 audible and silently mimed speech production. Previous strategies for neural decoding of

285 speech have primarily focused on direct classification of speech segments like phonemes

286 or words ${ }^{30,31,32,33}$. However, these demonstrations have been limited in their ability to

287 scale to larger vocabulary sizes and communication rates. Meanwhile, decoding of

288 auditory cortex responses has been more successful for continuous speech sounds ${ }^{18,34}$, in

289 part because of the direct relationship between the auditory encoding of spectrotemporal

290 information and the reconstructed spectrogram. An outstanding question has been

291 whether decoding vocal tract movements from the speech motor cortex could be used for

292 generating high-fidelity acoustic output.

We believe that cortical activity at vSMC electrodes was critical for decoding

294 (Figure 3e,f) because it encodes the underlying articulatory physiology that produces

295 speech $^{6}$. Our decoder explicitly incorporated this knowledge to simplify the complex

296 mapping from neural activity to sound by first decoding the physiological correlate of

297 neural activity and then transforming to speech acoustics. We have demonstrated that this

298 statistical mapping permits generalization with limited amounts of training. 
Direct speech synthesis has several major advantages over spelling-based

300 approaches. In addition to the capability to communicate at a natural speaking rate, it

301 captures prosodic elements of speech that are not available with text output, for example

302 pitch intonation (Figure $2 \mathrm{~d}$ ) and word emphasis ${ }^{35}$. Furthermore, a practical limitation for

303 current alternative communication devices is the cognitive effort required to learn and use

304 them. For patients in whom the cortical processing of articulation is still intact, a speech-

305 based BCI decoder may be far more intuitive and easier to learn to use $\mathrm{e}^{14,15}$.

306 Brain-computer interfaces are rapidly becoming clinically viable means to restore

307 lost function ${ }^{36}$. Impressive gains have already been made motor restoration of cursor

308 control and limb movements. Neural prosthetic control was first demonstrated in

309 participants without disabilities ${ }^{37,38,39}$ before translating the technology to participants

310 with tetraplegia ${ }^{40,41,42,43}$. While this articulatory-based approach establishes a new

311 foundation for speech decoding, we anticipate additional improvements from modeling

312 higher-order linguistic and planning goals ${ }^{44,45}$. Our results may be an important next step

313 in realizing speech restoration for patients with paralysis. 


\section{Methods}

320 Participants and experimental task. Three human participants (30 F, $31 \mathrm{~F}, 34 \mathrm{M})$

321 underwent chronic implantation of high-density, subdural electrode array over the lateral

322 surface of the brain as part of their clinical treatment of epilepsy (right, left, and right

323 hemisphere grids, respectively). Participants gave their written informed consent before

324 the day of the surgery. All participants were fluent in English. All protocols were

325 approved by the Committee on Human Research at UCSF. Each participant read and/or

326 freely spoke a variety of sentences. P1 read aloud two complete sets of 460 sentences

327 from the MOCHA-TIMIT database ${ }^{46}$. Additionally, $\mathrm{P} 1$ also read aloud passages from the

328 following stories: Sleeping Beauty, Frog Prince, Hare and the Tortoise, The Princess and

329 the Pea, and Alice in Wonderland. P2 read aloud one full set of 460 sentences from the

330 MOCHA-TIMIT database and further read a subset of 50 sentences an additional 9 times

331 each. P3 read 596 sentences describing three picture scenes and then freely described the

332 seen resulting in another 254 sentences. P3 also spoke 743 sentences during free response

333 interviews. In addition to audible speech, P1 also read 10 sentences 12 times each

334 alternating between audible and silent (mimed i.e. making the necessary mouth

335 movements) speech. Microphone recordings were obtained synchronously with the ECoG

336 recordings.

338 Data acquisition and signal processing. Electrocorticography was recorded with a

339 multi-channel amplifier optically connected to a digital signal processor (Tucker-Davis

340 Technologies). Speech was amplified digitally and recorded with a microphone 
341 simultaneously with the cortical recordings. ECoG electrodes were arranged in a 16 x 16

342 grid with $4 \mathrm{~mm}$ pitch. The grid placements were decided upon purely by clinical

343 considerations. ECoG signals were recorded at a sampling rate of 3,052 Hz. Each channel

344 was visually and quantitatively inspected for artifacts or excessive noise (typically $60 \mathrm{~Hz}$

345 line noise). The analytic amplitude of the high-gamma frequency component of the local

346 field potentials $(70-200 \mathrm{~Hz})$ was extracted with the Hilbert transform and down-sampled

347 to $200 \mathrm{~Hz}$. The low frequency component $(1-30 \mathrm{~Hz})$ was also extracted with a 5 th order

348 Butterworth bandpass filter and parallelly aligned with the high-gamma amplitude.

349 Finally, the signals were z-scored relative to a 30 second window of running mean and

350 standard deviation, so as to normalize the data across different recording sessions. We

351 studied high-gamma amplitude because it has been shown to correlate well with multi-

352 unit firing rates and has the temporal resolution to resolve fine articulatory movements ${ }^{17}$.

353 We also included a low frequency signal component due to the decoding performance

354 improvements note for reconstructing perceived speech from auditory cortex ${ }^{34}$. Decoding

355 models were constructed using all electrodes from vSMC, STG, and IFG except for

356 electrodes with bad signal quality as determined by visual inspection.

358 Phonetic and phonological transcription. For the collected speech acoustic recordings,

359 transcriptions were corrected manually at the word level so that the transcript reflected

360 the vocalization that the participant actually produced. Given sentence level

361 transcriptions and acoustic utterances chunked at the sentence level, hidden Markov

362 model based acoustic models were built for each participant so as to perform sub- 
363 phonetic alignment ${ }^{47}$. Phonological context features were also generated from the

364 phonetic labels, given their phonetic, syllabic and word contexts.

366 Cortical surface extraction and electrode visualization. We localized electrodes on

367 each individual's brain by co-registering the preoperative T1 MRI with a postoperative

368 CT scan containing the electrode locations, using a normalized mutual information

369 routine in SPM12. Pial surface reconstructions were created using Freesurfer. Final

370 anatomical labeling and plotting was performed using the img_pipe python package ${ }^{48}$.

371

372 Inference of articulatory kinematics. The articulatory kinematics inference model

373 comprises a stacked deep encoder-decoder, where the encoder combines phonological

374 and acoustic representations into a latent articulatory representation that is then decoded

375 to reconstruct the original acoustic signal. The latent representation is initialized with

376 inferred articulatory movement from Electromagnetic Midsagittal Articulography

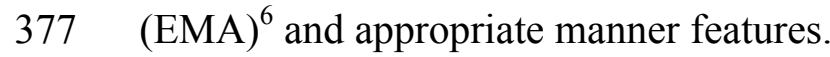

378 Chartier et al., 2018 described a statistical subject-independent approach to

379 acoustic-to-articulatory inversion which estimates 12 dimensional articulatory kinematic

380 trajectories ( $\mathrm{x}$ and y displacements of tongue dorsum, tongue blade, tongue tip, jaw,

381 upper lip and lower lip, as would be measured by EMA) using only the produced

382 acoustics and phonetic transcriptions. Since, EMA features do not describe all

383 acoustically consequential movements of the vocal tract, we append complementary

384 speech features that improve reconstruction of original speech. In addition to voicing and

385 intensity of the speech signal, we added place manner tuples (represented as continuous 
386 binary valued features) to bootstrap the EMA with what we determined were missing

387 physiological aspects in EMA. There were 18 additional values to capture the following

388 place-manner tuples: 1) velar stop, 2) velar nasal, 3) palatal approximant, 4) palatal

389 fricative, 5) palatal affricate, 6) labial stop, 7) labial approximant, 8) labial nasal, 9)

390 glottal fricative, 10) dental fricative, 11) labiodental fricative, 12) alveolar stop, 13)

391 alveolar approximant, 14) alveolar nasal, 15) alveolar lateral, 16) alveolar fricative, 17)

392 unconstructed, 18) voicing. For this purpose, we used an existing annotated speech

393 database (Wall Street Journal Corpus) ${ }^{49}$ and trained speaker independent deep recurrent

394 network regression models to predict these place-manner vectors only from the acoustics,

395 represented as 25-dimensional Mel Frequency Cepstral Coefficients (MFCCs). The

396 phonetic labels were used to determine the ground truth values for these labels (e.g., the

397 dimension "labial stop" would be 1 for all frames of speech that belong to the phonemes

$398 / \mathrm{p} /$, /b/ and so forth). However, with a regression output layer, predicted values were not

399 constrained to the binary nature of the input features. In all, these 32 combined feature

400 vectors form the initial articulatory feature estimates.

401 Finally, to ensure that the combined 32 dimensional representation has the

402 potential to reliably reconstruct speech, we designed an autoencoder to optimize these

403 values. Specifically, a recurrent neural network encoder is trained to convert

404 phonological and acoustic features to the initialized 32 articulatory representations and

405 then a decoder converts the articulatory representation back to the acoustics. The stacked

406 network is re-trained optimizing the joint loss on acoustic and EMA parameters. After

407 convergence, the encoder is used to estimate the final articulatory kinematic features that

408 act as the intermediate to decode acoustics from ECoG. 
410 Neural decoder. The decoder maps ECoG recordings to MFCCs via a two stage process

411 by learning intermediate mappings between ECoG recordings and articulatory kinematic

412 features, and between articulatory kinematic features and acoustic features. We

413 implemented this model using TensorFlow in python ${ }^{50}$. In the first stage, a stacked 3-

414 layer bLSTM ${ }^{16}$ learns the mapping between $300 \mathrm{~ms}$ windows of high-gamma and LFP

415 signals and the corresponding single time point of the 32 articulatory features. In the

416 second stage, an additional stacked 3-layer learns the mapping between the output of the

417 first stage (decoded articulatory features) and 32 acoustic parameters for full sentences

418 sequences. These parameters are are 25 dimensional MFCCs, 5 sub-band voicing

419 strengths for glottal excitation modelling, $\log (\mathrm{F} 0)$, voicing. At each stage, the model is

420 trained to with a learning rate of 0.001 to minimize mean-squared error of the target.

421 Dropout rate is set to $50 \%$ to suppress overfitting tendencies of the model. We use a

422 bLSTM because of their ability to retain temporally distant dependencies when

423 decoding a sequence ${ }^{51}$.

425 Speech synthesis from acoustic features. We used an implementation of the Mel-log

426 spectral approximation algorithm with mixed excitation ${ }^{22}$ to generate the speech

427 waveforms from estimates of the MFCCs from the neural decoder.

429 Model training procedure. As described, simultaneous recordings of ECoG and speech

430 are collected in short blocks of approximately 5 minutes. To partition the data for model

431 development, we allocated 2-3 blocks for model testing, 1 block for model optimization, 
432 and the remaining blocks for model training. The test sentences for P1 and P2 each

433 spanned 2 recording blocks and comprised 100 sentences read aloud. The test sentences

434 for P3 were different because the speech comprised 100 sentences over three blocks of

435 freely and spontaneously speech describing picture scenes.

436 For shuffling the data to test for significance, we shuffled the order of the

437 electrodes that were fed into the decoder. This method of shuffling preserved the

438 temporal structure of the neural activity.

440 Mel-Cepstral Distortion (MCD). To examine the quality of synthesized speech, we

441 calculated the Mel-Cepstral Distortion (MCD) of the synthesized speech when compared

442 the original ground-truth audio. MCD is an objective measure of error determined from

443 MFCCs and is correlated to subjective perceptual judgements of acoustic quality ${ }^{22}$. For

444 reference acoustic features $m c^{(y)}$ and decoded features $m c^{(\hat{y})}$,

$$
M C D=\frac{10}{\ln (10)} \sqrt{\sum_{0<d<25}\left(m c_{d}^{(y)}-m c_{d}^{(y)}\right)^{2}}
$$

447 Intelligibility Assessment. Listening tests using crowdsourcing are a standard way of

448 evaluating the perceptual quality of synthetic speech $^{52}$. We used the Amazon Mechanical

449 Turk to assess the intelligibility of the neurally synthesized speech samples. We set up a

450 listening task where naïve listeners identified which of 10 sentences was played in each

451 trial. A set of 60 sentences ( 6 trials of 10 unique sentences) were evaluated in this

452 assessment. These trials, also held out during training the decoder, were used in place of 
453 the 100 unique sentences tested throughout the rest of Figure 2 because the listeners

454 always had the same 10 sentences to chose from. Each trial sentence was listened to by

45550 different listeners. In all, 166 unique listeners took part in the evaluations.

457 Data limitation analysis. To assess the amount of training data affects decoder

458 performance, we partitioned the data by recording blocks and trained a separate model for 459 an allotted number of blocks. In total, 8 models were trained, each with one of the

460 following block allotments: [1, 2, 5, 10, 15, 20, 25, 28]. Each block comprised an average 461 of 50 sentences recorded in one continuous session.

463 Quantification of silent speech synthesis. By definition, there was no acoustic signal to 464 compare the decoded silent speech. In order to assess decoding performance, we

465 evaluated decoded silent speech in regards to the audible speech of the same sentence

466 uttered immediately prior to the silent trial. We did so by dynamically time warping ${ }^{53}$ the

467 decoded silent speech MFCCs to the MFCCs of the audible condition and computing

468 Pearson's correlation coefficient and Mel-cepstral distortion.

470 Phoneme acoustic similarity analysis. We compared the acoustic properties of decoded

471 phonemes to ground-truth to better understand the performance of our decoder. To do

472 this, we sliced all time points for which a given phoneme was being uttered and used the

473 corresponding time slices to estimate its distribution of spectral properties. With principal

474 components analysis (PCA), the 32 spectral features were projected onto the first 4

475 principal components before fitting the gaussian kernel density estimate (KDE) model. 
476 This process was repeated so that each phoneme had two KDEs representing either its

477 decoded and or ground-truth spectral properties. Using Kullback-Leibler divergence (KL

478 divergence), we compared each decoded phoneme KDE to every ground-truth phoneme

479 KDE, creating an analog to a confusion matrix used in discrete classification decoders.

480 KL divergence provides a metric of how similar two distributions are to one another by

481 calculating how much information is lost when we approximate one distribution with

482 another. Lastly, we used Ward's method for agglomerative hierarchical clustering to

483 organize the phoneme similarity matrix.

484

485

486

487

488

489 


\section{References:}

2. Herff, C., \& Schultz, T. (2016). Automatic Speech Recognition from Neural

1. Wolpaw, J. R., Birbaumer, N., McFarland, D. J., Pfurtscheller, G., \& Vaughan, T. M. (2002). Brain-computer interfaces for communication and control. Clinical neurophysiology, 113(6), 767-791.

$$
\text { Signals : A Focused Review, 10(September), 1-7. }
$$

https://doi.org/10.3389/fnins.2016.00429

3. Bocquelet F, Hueber T, Girin L, Chabardès S, Yvert B. (2016). Key considerations in designing a speech brain computer interface. J Physiol Paris, 110: 392-401.

4. Brumberg, J. S., Pitt, K. M., Mantie-Kozlowski, A., \& Burnison, J. D. (2018). Brain-Computer Interfaces for Augmentative and Alternative Communication: A Tutorial. American journal of speech-language pathology, 27(1), 1-12.

5. Lotte, F., Brumberg, J. S., Brunner, P., Gunduz, A., Ritaccio, A. L., Guan, C., \& Schalk, G. (2015). Electrocorticographic representations of segmental features in continuous speech. Frontiers in human neuroscience, 9, 97.

6. Chartier, J., Anumanchipalli, G. K., Johnson, K., \& Chang, E. F. (2018). Encoding of Articulatory Kinematic Trajectories in Human Speech Sensorimotor Cortex. Neuron, 98(5), 1042-1054.e4. https://doi.org/10.1016/j.neuron.2018.04.031

7. Majaranta, P., \& Räihä, K. J. (2002, March). Twenty years of eye typing: systems and design issues. In Proceedings of the 2002 symposium on Eye tracking research \& applications (pp. 15-22). ACM. mental prosthesis utilizing event-related brain potentials. Electroencephalography and clinical Neurophysiology, 70(6), 510-523.

9. Pandarinath, C., Nuyujukian, P., Blabe, C. H., Sorice, B. L., Saab, J., Willett, F. R., ... Henderson, J. M. (2017). High performance communication by people with paralysis using an intracortical brain-computer interface. ELife, 6, 1-27. https://doi.org/10.7554/eLife.18554

10. Guenther, F. H., Brumberg, J. S., Joseph Wright, E., Nieto-Castanon, A., Tourville, J. A., Panko, M., ... Kennedy, P. R. (2009). A wireless brain-machine interface for real-time speech synthesis. PLoS ONE, 4(12). https://doi.org/10.1371/journal.pone.0008218 
11. Newell, A., Langer, S., \& Hickey, M. (1998). The rôle of natural language processing in alternative and augmentative communication. Natural Language Engineering, 4(1), 1-16.

528

12. Bocquelet, F., Hueber, T., Girin, L., Savariaux, C., \& Yvert, B. (2016). Real-time control of an articulatory-based speech synthesizer for brain computer interfaces. PLoS computational biology, 12(11), e1005119.

13. Browman, C. P., \& Goldstein, L. (1992). Articulatory phonology: An overview. Phonetica, 49(3-4), 155-180.

14. Sadtler, P. T., Quick, K. M., Golub, M. D., Chase, S. M., Ryu, S. I., Tyler-Kabara, E. C., ... \& Batista, A. P. (2014). Neural constraints on learning. Nature, 512(7515), 423.

15. Golub, M. D., Sadtler, P. T., Oby, E. R., Quick, K. M., Ryu, S. I., Tyler-Kabara, E. C., ... \& Yu, B. M. (2018). Learning by neural reassociation. Nat. Neurosci., 21.

16. Graves, A., \& Schmidhuber, J. (2005). Framewise phoneme classification with bidirectional LSTM and other neural network architectures. Neural Networks, 18(5-6), 602-610.

17. Crone, N.E., Hao, L., Hart, J., Jr., Boatman, D., Lesser, R.P., Irizarry, R., and Gordon, B. (2001). Electrocorticographic gamma activity during word production in spoken and sign language. Neurology 57, 2045-2053.

18. Akbari H., Khalighinejad B., Herrero J., Mehta A., Mesgarani N. (2018) Reconstructing intelligible speech from the human auditory cortex. bioRxiv 350124; doi: https://doi.org/10.1101/350124

19. Bouchard, K.E., Mesgarani, N., Johnson, K., and Chang, E.F. (2013). Functional organization of human sensorimotor cortex for speech articulation. Nature 495, $327-332$.

20. Mesgarani, N., Cheung, C., Johnson, K., \& Chang, E. F. (2014). Phonetic feature encoding in human superior temporal gyrus. Science, 343(6174), 1006-1010.

21. Flinker, A., Korzeniewska, A., Shestyuk, A. Y., Franaszczuk, P. J., Dronkers, N. F., Knight, R. T., \& Crone, N. E. (2015). Redefining the role of Broca's area in speech. Proceedings of the National Academy of Sciences, 112(9), 2871-2875.

22. Zheng, F., Zhang, G., \& Song, Z. (2001). Comparison of different implementations of MFCC. Journal of Computer science and Technology, 16(6), 582-589. 
23. Yoshimura, T., Tokuda, K., Masuko, T., Kobayashi, T., \& Kitamura, T., (2001). Mixed excitation for HMM-based Speech Synthesis, Eurospeech 2001.

24. Davis, S. B., \& Mermelstein, P. (1990). Comparison of parametric representations for monosyllabic word recognition in continuously spoken sentences. In Readings in speech recognition (pp. 65-74).

25. Janke, M. and Diener, L. (2017). EMG-to-Speech: Direct Generation of Speech From Facial Electromyographic Signals. IEEE/ACM Trans. Audio, Speech and Lang. Proc. 25, 12 (December 2017), 2375-2385. DOI: https://doi.org/10.1109/TASLP.2017.2738568

26. Drullman, R., Festen, J. M. \& Plomp, R. Effect of temporal envelope smearing on speech reception. J. Acoust. Soc. Am. 95, 1053-1064 (1994).

27. Shen, Jonathan et. al., (2018) Natural TTS by conditioning Wavenet on Melspectrogram predictions. In proceedings of ICASSP 2018, https://arxiv.org/abs/1712.05884

28. Kominek, J., Schultz, T., and Black, A. (2008). "Synthesizer voice quality of new languages calibrated with mean mel cepstral distortion", In SLTU-2008, 63-68.

29. Janke, M. (2016). EMG-to-Speech: Direct Generation of Speech from facial Electromyographic Signals. PhD Dissertation, Karlshruhe Institute of Technology, Germany, 2016

30. Mugler, E.M., Patton, J.L., Flint, R.D., Wright, Z.A., Schuele, S.U., Rosenow, J., Shih, J.J., Krusienski, D.J., and Slutzky, M.W. (2014). Direct classification of all American English phonemes using signals from functional speech motor cortex. J. Neural Eng. 11, 035015.

31. Herff, C., Heger, D., de Pesters, A., Telaar, D., Brunner, P., Schalk, G., and Schultz, T. (2015). Brain-to-text: decoding spoken phrases from phone representations in the brain. speech recognition: continuous phoneme decoding using spatiotemporal representations of human cortical activity. Journal of neural engineering, 13(5), 056004. for neural data analysis: speech classification and cross-frequency coupling in human sensorimotor cortex. arXiv preprint arXiv:1803.09807. 
34. Pasley, B. N., David, S. V, Mesgarani, N., Flinker, A., \& Shamma, S. A. (2012). Reconstructing Speech from Human Auditory Cortex. PLoS Biol, 10(1), 1001251. https://doi.org/10.1371/journal.pbio.1001251

35. Dichter B. K., Breshears J. D., Leonard M. K., and Chang E. F. (2018) The Control of Vocal Pitch in Human Laryngeal Motor Cortex. Cell, 174, 21-31

36. Leuthardt, E. C., Schalk, G., Moran, D., \& Ojemann, J. G. (2006). The emerging world of motor neuroprosthetics: a neurosurgical perspective. Neurosurgery, 59(1), 1.

37. Wessberg J, Stambaugh CR, Kralik JD, Beck PD, Laubach M, et al. (2000) Realtime prediction of hand trajectory by ensembles of cortical neurons in primates. Nature 408: 361-365.

38. Serruya MD, Hatsopoulos NG, Paninski L, Fellows MR, Donoghue JP (2002) Instant neural control of a movement signal. Nature 416: 141-142.

39. Taylor DM, Tillery SI, Schwartz AB (2002) Direct cortical control of 3D neuroprosthetic devices. Science 296: 1829-1832.

40. Hochberg, L. R., Serruya, M. D., Friehs, G. M., Mukand, J. A., Saleh, M., Caplan, A. H., ... \& Donoghue, J. P. (2006). Neuronal ensemble control of prosthetic devices by a human with tetraplegia. Nature, 442(7099), 164

41. Collinger, J. L., Wodlinger, B., Downey, J. E., Wang, W., Tyler-Kabara, E. C., Weber, D. J., ... \& Schwartz, A. B. (2013). High-performance neuroprosthetic control by an individual with tetraplegia. The Lancet, 381(9866), 557-564.

42. Aflalo, T., Kellis, S., Klaes, C., Lee, B., Shi, Y., Pejsa, K., ... \& Andersen R. A. (2015). Decoding motor imagery from the posterior parietal cortex of a tetraplegic human. Science, 348(6237), 906-910. Miller, J. P., ... \& Peckham, P. H. (2017). Restoration of reaching and grasping movements through brain-controlled muscle stimulation in a person with tetraplegia: a proof-of-concept demonstration. The Lancet, 389(10081), 1821posterior parietal cortex, Nature 386, 167-170, 1997 
45. Huth, A. G., de Heer, W. A., Griffiths, T. L., Theunissen, F. E., \& Gallant, J. L. (2016). Natural speech reveals the semantic maps that tile human cerebral cortex. Nature, 532(7600), 453.

$626 \quad$ Method References

46. Wrench, A. (1999). MOCHA: multichannel articulatory database. http://www.cstr.ed.ac.uk/research/projects/artic/mocha.html.

47. Prahallad, K., Black, A.W., and Mosur, R. (2006). Sub-phonetic modeling for capturing pronunciation variations for conversational speech synthesis. In Proceedings of the 2006 IEEE International Conference on Acoustics Speech and Signal Processing (ICASSP), pp. I-I.

48. Hamilton, L. S., Chang, D. L., Lee, M. B., \& Chang, E. F. (2017). Semiautomated Anatomical Labeling and Inter-subject Warping of High-Density Intracranial Recording Electrodes in Electrocorticography. Frontiers in Neuroinformatics, 11, 62. http://doi.org/10.3389/fninf.2017.00062

49. Paul, B., D, and Baker, M., J, (1992). The design for the wall street journal-based CSR corpus. In Proceedings of the workshop on Speech and Natural Language (HLT '91). Association for Computational Linguistics, Stroudsburg, PA, USA, 357-362. DOI: https://doi.org/10.3115/1075527.1075614

50. Martín Abadi, Ashish Agarwal, Paul Barham, Eugene Brevdo, Zhifeng Chen, et al. (2015). TensorFlow: Large-scale machine learning on heterogeneous systems. http://www.tensorflow.org

51. Hochreiter, S., and Schmidhuber, J. (1997). Long short-term memory. Neural Comput. 9, 1735-1780.

52. Wolters, M. K., Isaac, Renals, S., Evaluating Speech Synthesis intelligibility using Amazon Mechanical Turk. (2010) In proceedings of ISCA speech synthesis workshop (SSW7), 2010.

649 53. Berndt, D. J., \& Clifford, J. (1994). Using dynamic time warping to find patterns in time series. In KDD workshop (Vol. 10, No. 16, pp. 359-370). 


\section{Extended Data:}
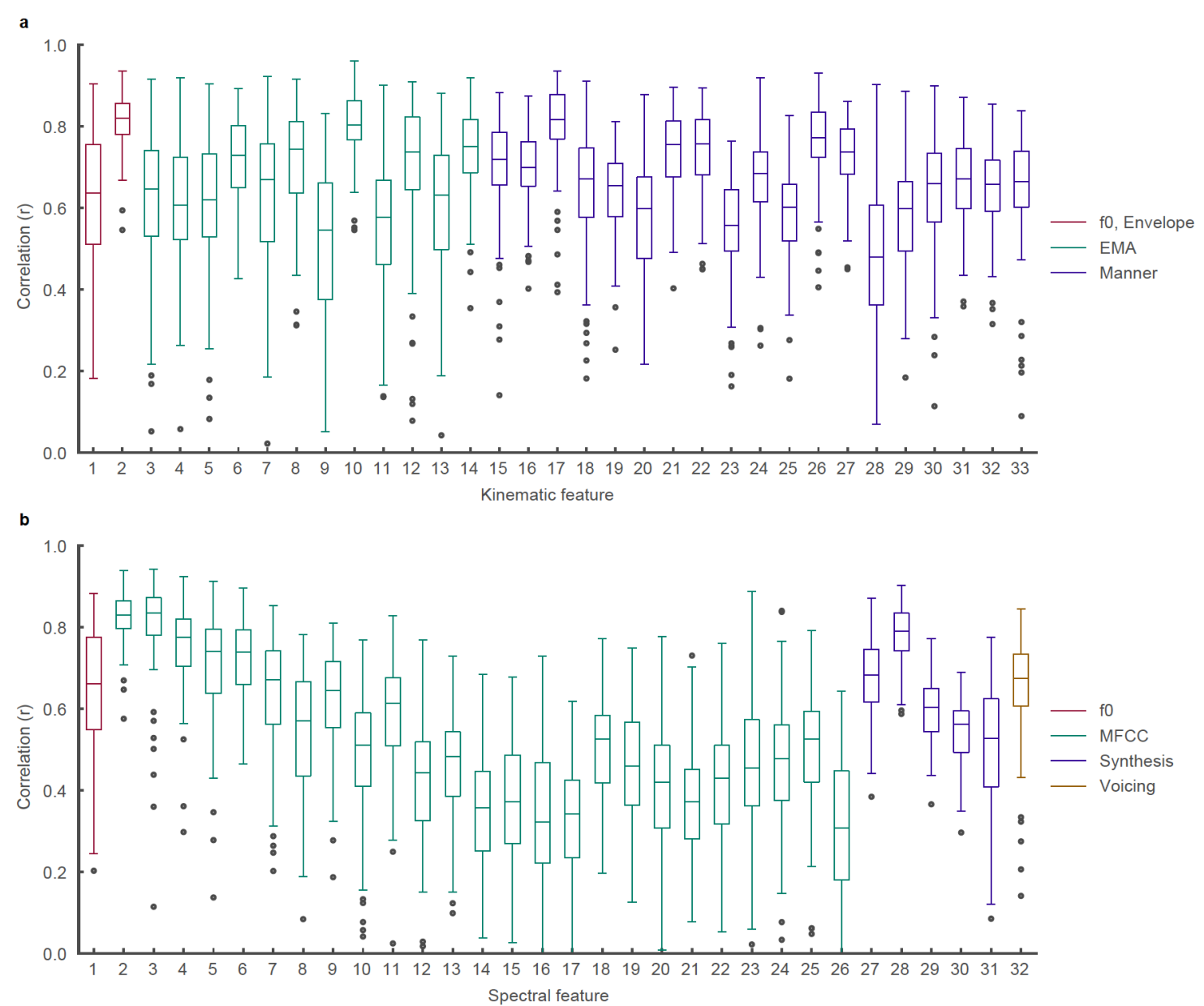

656 Extended Data Figure 1: Decoding performance of kinematic and spectral features.

657 a, Correlations of all 33 decoded articulatory kinematic features with ground-truth. EMA

658 features represent $\mathrm{X}$ and $\mathrm{Y}$ coordinate traces of articulators (lips, jaw, and three points of

659 the tongue) along the midsagittal plane of the vocal tract. Manner features represent

660 complementary kinematic features to EMA that further describe acoustically

661 consequential movements. b, Correlations of all 32 decoded spectral features with

662 ground-truth. MFCC features are 25 mel-frequency cepstral coefficients that describe

663 power in perceptually relevant frequency bands. Synthesis features describe glottal

664 excitation weights necessary for speech synthesis. 


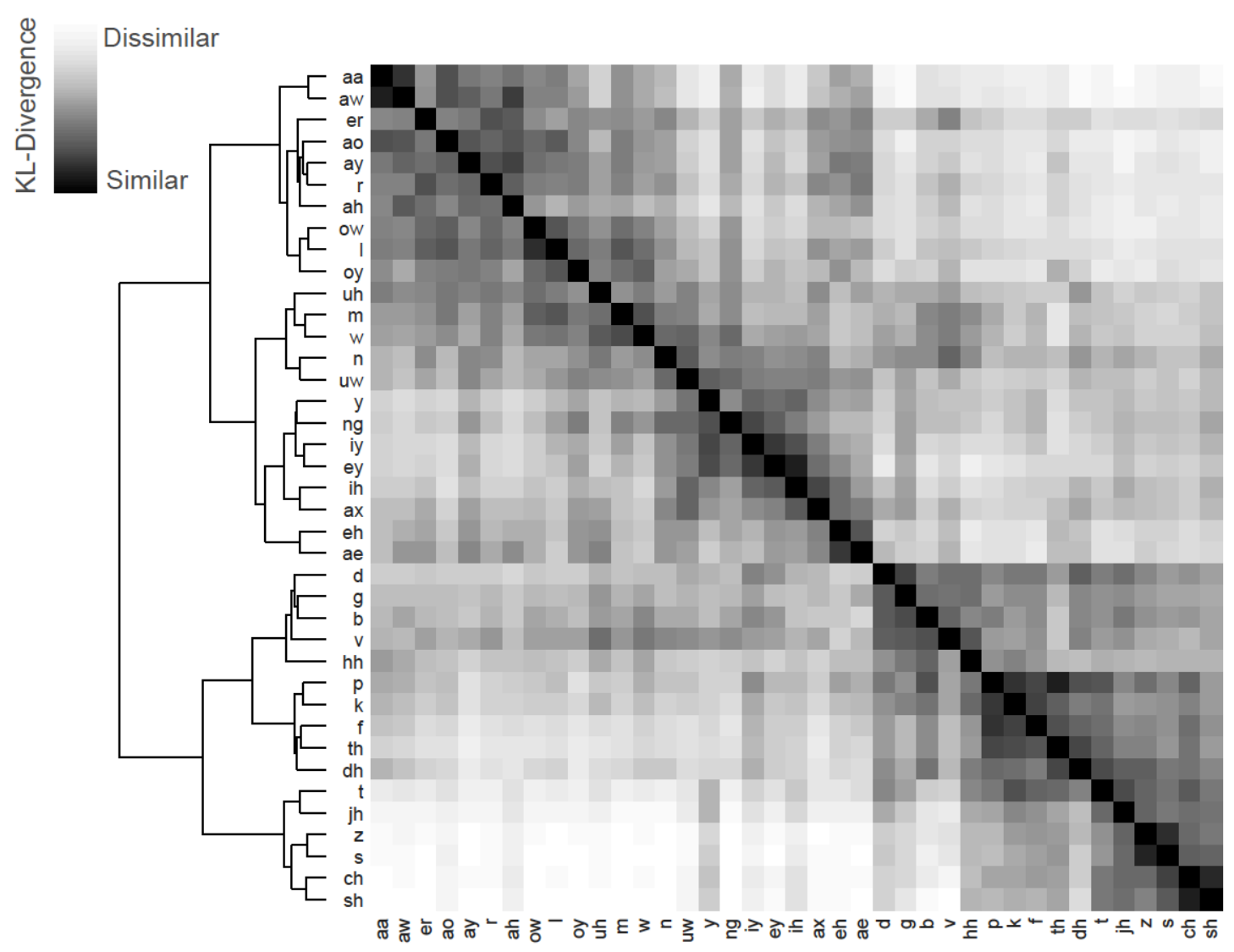

666 Extended Data Figure 2: Ground-truth acoustic similarity matrix. Compares acoustic

667 properties of ground-truth spoken phonemes with one another. Similarity is computed by

668 first estimating a gaussian kernel density for each phoneme and then computing the

669 Kullback-Leibler (KL) divergence between a pair of a phoneme distributions. Each row

670 compares the acoustic properties of a two ground-truth spoken phonemes. Hierarchical

671 clustering was performed on the resulting similarity matrix.

\section{Acknowledgments}

674 We thank Matthew Leonard, Neal Fox for their helpful comments on the manuscript. We

675 also thank Ben Speidel for his work reconstructing MRI images of patients' brains. This 
676 work was supported by grants from the NIH (DP2 OD008627 and U01 NS098971-01).

677 E.F.C is a New York Stem Cell Foundation- Robertson Investigator. This research was

678 also supported by The New York Stem Cell Foundation, the Howard Hughes Medical

679 Institute, The McKnight Foundation, The Shurl and Kay Curci Foundation, and The

680 William K. Bowes Foundation.

681

682 Author Contributions Conception G.K.A., J.C., and E.F.C.; Articulatory kinematics

683 inference G.K.A; Decoder design G.K.A and J.C.; Decoder analyses: J.C.; Data

684 collection G.K.A., E.F.C., and J.C.; Prepared manuscript all; Project Supervision E.F.C.

685

686 